\title{
The derivation of an isotropic model for internal waves and its application to wave generation
}

\author{
C. Yuan ${ }^{\mathrm{a}}$, Z. Wang ${ }^{\mathrm{b}, \mathrm{c}, *}, \mathrm{X}$. Chen ${ }^{\mathrm{a}, \mathrm{d}}$ \\ a School of Mathematical Sciences, Ocean University of China, Qingdao 266100, China \\ ${ }^{\mathrm{b}}$ Key laboratory for Mechanics in Fluid Solid Coupling Systems, Institute of Mechanics, Chinese Academy of Sciences, Beijing 100190, China \\ c School of Engineering Science, University of Chinese Academy of Sciences, Beijing 100049, China \\ ${ }^{\mathrm{d}}$ Hubei Key Laboratory of Marine Geological Resources, China University of Geosciences, Wuhan 430074, China
}

\section{A R T I C L E I N F O}

\section{Keywords:}

3D Internal waves

Benney-Luke equation

Isotropic model

\begin{abstract}
A B S T R A C T
In contrast to two-dimensional oceanic internal waves which have been extensively investigated, there are relatively few theoretical studies on three-dimensional internal waves. The most remarkable theory describing three-dimensional internal waves is the Kadomtsev-Petviashvili (KP) equation. Nevertheless, two shortcomings - unidirectional propagation and anisotropy - limit its application in some general cases. In the current paper, via an asymptotic analysis, we derive an isotropic and bidirectional model, the modified Benney-Luke equation, for a two-layer fluid with bottom topography which can vary both in time and space, therefore tides and currents can be incorporated into the internal wave problem due to the relative motion. In the derivation, the assumption of incompressibility and long waves are invoked and the effects of the Earth's rotation and dissipation are ignored. Based on this model, the generation of nonlinear internal waves by background currents and barotropic tides flowing over topography are investigated. The resulting equation aims to facilitate dynamical analyses, as well as the interpretation of in-situ observational data and laboratory experimental results.
\end{abstract}

\section{Introduction}

The importance of internal waves in the ocean has been recognized for the last several decades in part because of the development of modern observational techniques. Internal waves have a peculiar feature manifested as large vertical fluctuations in the interior of the water column accompanied by much smaller sea surface displacements. The strong horizontal currents associated with the waves can produce perceptible responses at the ocean's surface recorded by orbiting satellites. Given the relative weakness of the buoyancy (at the order of $10^{-3}$ of gravity) acting as the restoring force, internal waves usually possess large amplitudes. Considering the inconspicuousness at the surface, such waves can pose potential threats to ocean structures and underwater vehicles. In addition, internal waves also play a crucial role in the marginal marine ecosystem and global energy budget, see Kunze (2017) and Woodson (2018) and the references therein. Based on in-situ observations and laboratory experiments, a large amount of research has been conducted (see the review by Helfrich and Melville, 2006), among which theoretical analyses provide a deep and essential understanding of this subject.

In the context of nonlinear internal waves, Benney (1966) and Benjamin (1966) provided among the first derivations of the celebrated
Korteweg-de Vries (KdV) equation for the description of unidirectional weakly nonlinear long waves. Importantly, Osborne and Burch (1980) provided a paradigm for the implementation of the KdV equation to model observed internal solitary waves. Thereafter, the research of internal waves based on the KdV equation prospered and several variants emerged, including the extended KdV equation incorporating an additional cubic nonlinear term (Lee and Beardsley, 1974; Djordjevic and Redekopp, 1978; Grimshaw et al., 2002), the rotation-modified $\mathrm{KdV}$ equation including the Earth's rotation (Ostrovsky, 1978; Grimshaw, 1985), a modified KdV equation taking bottom friction into account (Grimshaw, 2002; Grimshaw et al., 2003), a modified KdV equation including the effect of background shear, slowly varying depth, and continuous stratification (Grimshaw, 1981; Zhou and Grimshaw, 1989; Holloway et al., 1997), and the twodimensional version of the $\mathrm{KdV}$ equation, namely the well-known Kadomtsev-Petviashvili (KP) equation (Kadomtsev and Petviashvili, 1970; Grimshaw, 1981). Nonetheless, KdV-type equation is only applied to cases for which the wavelength is large with respect to the depth, i.e. the shallow-water regime. To mitigate this shortcoming, the weakly nonlinear Benjamin-Ono (BO) equation was derived for

* Corresponding author at: Key laboratory for Mechanics in Fluid Solid Coupling Systems, Institute of Mechanics, Chinese Academy of Sciences, Beijing 100190, China.

E-mail address: zwang@imech.ac.cn (Z. Wang). 
infinitely deep fluids (Benjamin, 1966; Ono, 1975) and the Intermediate Long Wave (ILW) equation for intermediate depth (Joseph, 1977; Kubota et al., 1978). As pointed out by Ostrovsky and Stepanyants (2005), the BO and ILW equations have narrower ranges of validity and fail to make good predictions beyond these ranges. They also cautioned that the interpretation of observational data using the $\mathrm{BO}$ and ILW equations should be given much more care, although Wang and Pawlowicz (2011) achieved a satisfactory result with the BO equation while considering large-amplitude internal waves in deep water.

Although most of the aforementioned equations have been successfully and widely used to study the properties of solitary wave solutions as well as to interpret data from in-situ measurements and laboratory experiments, the weakly nonlinear assumption limits their applications especially when large-amplitude waves are involved. As a useful extension of the extended KdV equation (with the cubic nonlinear term), the Miyata-Choi-Camassa (MCC) equations with full nonlinearity and weak dispersion were derived by Miyata $(1985,1988)$ and Choi and Camassa (1999). The MCC equations are bidirectional (cf. the unidirectional feature of the $\mathrm{KdV}$ equation), and Choi and Camassa (1999) illustrated very good agreement with results from both laboratory experiments and numerical solutions of the Euler equations. Later on, Ostrovsky and Grue (2003) obtained unidirectional evolution equations taking nonlinear dispersive effects into account. These equations generally agree well with the MCC equations and further preclude the intrinsic Kelvin-Helmholtz instability at high wavenumbers in the MCC equations. Nevertheless, a more accurate way to investigate solitary waves is in the framework of the full Euler equations without assumptions of weak nonlinearity or/and weak dispersion. The DubreilJacotin-Long (DJL) equation describes the streamline displacement in the form of a strongly nonlinear elliptic partial differential equation, which amounts to the full set of stratified Euler equations, see the early references by Long (1955) and later by Benjamin (1966) and Tung et al. (1982). A further extension of the DJL equation taking the effect of background shear into consideration was proposed by Stastna and Lamb (2002) and Lamb (2003). Note that the DJL equation is time-independent and incapable of examining wave evolutions. The interested reader is referred to a more comprehensive review by Helfrich and Melville (2006).

Previous theoretical work has been primarily focused on internal waves in one horizontal dimension, albeit we note that Pierini (1989), Cai and Xie (2010), Yuan et al. (2018a) and Yuan et al. (2018b) conducted research based on the KP equation. However, the KP equation is unidirectional and, moreover, it assumes that the variations in the transverse direction are one-order smaller than those in the wave propagation direction indicating that it is an inappropriate model for general cases where the variations are comparable along perpendicular axes. It concludes that isotropic models for internal waves are of interest. Therefore, in this paper, we derive an isotropic bidirectional model equation in two horizontal dimensions based on the assumption of weak nonlinearity. The derivation is provided in Section 2, together with the associated numerical method. To check the capability of the derived equation, several numerical experiments are carried out in Section 3. The cases of constant background current passing over topography are illustrated, which can be further categorized as subcritical, critical and supercritical scenarios according to the competition of strength between background current and intrinsic linear wave phase speed, while the generation processes excited by barotropic tides flowing in two perpendicular directions are also demonstrated. Finally, we give concluding remarks in Section 4 .

\section{The modified Benney-Luke equation}

\subsection{Model setup}

We consider three-dimensional incompressible, inviscid and irrotational flows, composed of two immiscible layers with a lighter one

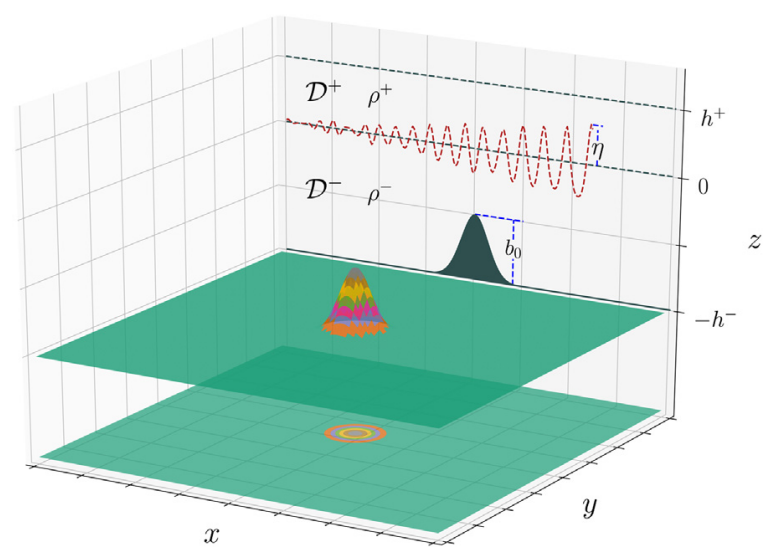

Fig. 1. The schematic representation of two-layer fluid characterized by densities $\rho^{+}, \rho^{-}$and thicknesses $h^{+}, h^{-}$. A moving Gaussian topography mimicking background current $U(t)$ and $V(t)$ passing over topography is given by $b=$ $b_{0} \exp \left\{-\left[(x-U t) / x_{w}\right]^{2}-\left[(y-V t) / y_{w}\right]^{2}\right\}$.

lying above a heavier one. The two fluids are separated by a sharp interface $z=\eta(x, y, t)$, where $x, y$ are horizontal coordinates and the $z$-axis points upwards with $z=0$ the undisturbed interface. The upper layer (denoted by $\mathcal{D}^{+}$) is bounded above by a rigid lid located at $z=h^{+}$, and the lower layer (denoted by $D^{-}$) is bounded below by a bottom topography $z=-h^{-}+b(x, y, t)$ varying in space and time where $b(x, y, t)$ is a prescribed function. The density of the fluid in each layer is subject to be constant designated by $\rho^{+}$and $\rho^{-}$corresponding to $D^{+}$and $D^{-}$ respectively, where $\rho^{+}<\rho^{-}$as in the realistic ocean (see Fig. 1).

The velocity potential $\phi(x, y, z)$ in each layer satisfies Laplace's equation,

$\Delta \phi^{-}+\phi_{z z}^{-}=0, \quad$ for $-h^{-}+b(x, y, t)<z<\eta(x, y, t)$

$\Delta \phi^{+}+\phi_{z z}^{+}=0, \quad$ for $\eta(x, y, t)<z<h^{+}$

where $\Delta=\partial_{x x}+\partial_{y y}$ is the two-dimensional Laplace operator in the horizontal variables. On the interface $z=\eta(x, y, t)$, the nonlinear kinematic and dynamic boundary conditions read, respectively, as follows

$\eta_{t}=\phi_{z}^{-}-\nabla \phi^{-} \cdot \nabla \eta=\phi_{z}^{+}-\nabla \phi^{+} \cdot \nabla \eta$,

$\rho^{-} \phi_{t}^{-}-\rho^{+} \phi_{t}^{+}+\frac{\rho^{-}}{2}\left[\left|\nabla \phi^{-}\right|^{2}+\left(\phi_{z}^{-}\right)^{2}\right]-$

$\frac{\rho^{+}}{2}\left[\left|\nabla \phi^{+}\right|^{2}+\left(\phi_{z}^{+}\right)^{2}\right]+\left(\rho^{-}-\rho^{+}\right) g \eta=0$,

where $\nabla$ and $\nabla$. are the respective gradient and divergent operator in the horizontal variables, and $g$ is the acceleration due to gravity. The kinematic boundary condition (1) implies that the normal velocity is continuous across the interface

$\left(\phi_{x}^{-}, \phi_{y}^{-}, \phi_{z}^{-}\right)^{\top} \cdot \mathbf{n}=\left(\phi_{x}^{+}, \phi_{y}^{+}, \phi_{z}^{+}\right)^{\top} \cdot \mathbf{n}$

where $\mathbf{n}=\left(-\eta_{x},-\eta_{y}, 1\right)^{\top} / \sqrt{1+|\nabla \eta|^{2}}$ is the unit normal vector pointing upwards on the interface. Finally, the boundary conditions

$$
\begin{aligned}
\phi_{z}^{+}=0, & \text { at } z=h^{+}, \\
\phi_{z}^{-}-\nabla b \cdot \nabla \phi^{-}=b_{t}, & \text { at } z=-h^{-}+b(x, y, t),
\end{aligned}
$$

complete the whole system.

\subsection{Derivation}

Recently, Ablowitz et al. (2006) introduced an explicit non-local formulation for the classical water-wave problem in both two and three dimensions. It was then generalized to study interfacial fluids with a free surface by Haut and Ablowitz (2009) and to include timedependent bottom topography by Curtis and Shen (2015). Stemming from these work, the derivation of the desired modified Benney-Luke 
equation for internal waves is achieved as shown in the subsequent analyses.

Following Ablowitz et al. (2006), it is straightforward to verify that the identity

$\left(\phi_{z} \psi_{x}+\phi_{x} \psi_{z}\right)_{x}+\left(\phi_{z} \psi_{y}+\phi_{y} \psi_{z}\right)_{y}+\left(\phi_{z} \psi_{z}-\phi_{x} \psi_{x}-\phi_{y} \psi_{y}\right)_{z}=0$

holds for an arbitrary harmonic function $\psi(x, y, z)$, where $\phi(x, y, z)$ are the aforementioned velocity potentials in both layers and the subscripts denote the partial derivative with respect to corresponding variables. Applying the Divergence Theorem to Eq. (6) in the lower layer $\mathcal{D}^{-}$, one obtains

$0=\int\left[-\eta_{x}\left(\phi_{z}^{-} \psi_{x}+\phi_{x}^{-} \psi_{z}\right)-\eta_{y}\left(\phi_{z}^{-} \psi_{y}+\phi_{y}^{-} \psi_{z}\right)+\left(\phi_{z}^{-} \psi_{z}-\nabla \phi^{-} \cdot \nabla \psi\right)\right]_{z=\eta} d \mathbf{r}$

$+\int\left[b_{x}\left(\phi_{z}^{-} \psi_{x}+\phi_{x}^{-} \psi_{z}\right)+b_{y}\left(\phi_{z}^{-} \psi_{y}+\phi_{y}^{-} \psi_{z}\right)-\left(\phi_{z}^{-} \psi_{z}-\nabla \phi^{-} \cdot \nabla \psi\right)\right]_{z=-h^{-}+b} d \mathbf{r}$

where $\mathbf{r}=(x, y)$ is a vector in the horizontal plane. Substituting $\psi=$ $e^{\mathrm{ik} \cdot \mathbf{r}+k z}$ into Eq. (7) yields

$0=\int e^{\mathrm{i} \mathbf{k} \cdot \mathbf{r}+k\left(\eta+h^{-}\right)}\left[k \eta_{t}-\mathbf{i} \mathbf{k} \cdot \nabla \boldsymbol{\Phi}^{-}\right] d \mathbf{r}+\int e^{\mathrm{i} \mathbf{k} \cdot \mathbf{r}+k b}\left[-k b_{t}+\mathbf{i} \mathbf{k} \cdot \nabla \boldsymbol{\Phi}^{b}\right] d \mathbf{r}$,

where $\mathbf{k}=\left(k_{x}, k_{y}\right)$ is the wavenumber vector, $k=\left(k_{x}^{2}+k_{y}^{2}\right)^{1 / 2}$ is its modulus, $\Phi^{-}=\phi^{-}(x, y, \eta, t)$, and $\Phi^{b}=\phi^{-}(x, y, b, t)$. Since $e^{i \mathbf{k} \cdot \mathbf{r}-k z}$ is also a solution to Laplace's equation, a substitution into (7) then leads to

$0=\int e^{\mathrm{i} \mathbf{k} \cdot \mathbf{r}-k\left(\eta+h^{-}\right)}\left[-k \eta_{t}-\mathrm{i} \mathbf{k} \cdot \nabla \boldsymbol{\Phi}^{-}\right] d \mathbf{r}+\int e^{\mathrm{i} \mathbf{k} \cdot \mathbf{r}-k b}\left[k b_{t}+\mathbf{i} \mathbf{k} \cdot \nabla \boldsymbol{\Phi}^{b}\right] d \mathbf{r}$.

Adding and subtracting (8) and (9) yield, respectively,

$$
\begin{aligned}
& 0=\int e^{\mathrm{i} \mathbf{k} \cdot \mathbf{r}}[\left.k \sinh k\left(\eta+h^{-}\right) \eta_{t}-\mathbf{i k} \cdot \nabla \Phi^{-} \cosh k\left(\eta+h^{-}\right)\right] d \mathbf{r} \\
&+\int e^{\mathbf{i k} \cdot \mathbf{r}}\left[-k \sinh (k b) b_{t}+\mathbf{i k} \cdot \nabla \Phi^{b} \cosh (k b)\right] d \mathbf{r}
\end{aligned}
$$

and

$$
\begin{aligned}
& 0=\int e^{\mathbf{i k} \cdot \mathbf{r}} {\left[\cosh k\left(\eta+h^{-}\right) \eta_{t}-\mathbf{i k} \cdot \nabla \Phi^{-} \frac{\sinh k\left(\eta+h^{-}\right)}{k}\right] d \mathbf{r} } \\
&+\int e^{\mathrm{i} \mathbf{k} \cdot \mathbf{r}}\left[-\cosh (k b) b_{t}+\mathbf{i k} \cdot \nabla \Phi^{b} \frac{\sinh (k b)}{k}\right] d \mathbf{r} .
\end{aligned}
$$

In the same vein, one can obtain the global relation in the upper layer $\mathcal{D}^{+}$,

$0=\int e^{\mathbf{i k} \cdot \mathbf{r}}\left[\cosh k\left(h^{+}-\eta\right) \eta_{t}+\mathbf{i k} \cdot \nabla \Phi^{+} \frac{\sinh k\left(h^{+}-\eta\right)}{k}\right] d \mathbf{r}$,

where $\Phi^{+}=\phi^{+}(x, y, \eta, t)$. Furthermore, if the density ratio of the upper and lower layer is denoted by $\sigma=\rho^{+} / \rho^{-}<1$, then the dynamic boundary condition on the interface can be rewritten as

$$
\begin{gathered}
\left(\Phi^{-}-\sigma \Phi^{+}\right)_{t}+(1-\sigma) g \eta+\frac{1}{2}\left|\nabla \Phi^{-}\right|^{2}-\frac{\sigma}{2}\left|\nabla \Phi^{+}\right|^{2} \\
-\frac{1}{2} \frac{\left(\eta_{t}+\nabla \eta \cdot \nabla \Phi^{-}\right)^{2}}{1+|\nabla \eta|^{2}}+\frac{\sigma}{2} \frac{\left(\eta_{t}+\nabla \eta \cdot \nabla \Phi^{+}\right)^{2}}{1+|\nabla \eta|^{2}}=0 .
\end{gathered}
$$

The Boussinesq scaling is introduced through the typical length $L$ and wave amplitude $a$ :

$x, y \sim L, \quad \eta \sim a, \quad t \sim \frac{L}{\sqrt{g h^{-}(1-\sigma)}}, \quad \Phi^{ \pm, b} \sim \frac{a g L \sqrt{1-\sigma}}{\sqrt{g h^{-}}}, \quad b \sim \frac{a^{2}}{h^{-}}$.

Small parameters $\epsilon=a / h^{-}$and $\mu=h^{-} / L$ are defined to measure the nonlinearity and dispersion respectively, and the ratio of the upper layer to lower layer thickness $\tau=h^{+} / h^{-}$is assumed to be of order one. After non-dimensionalization using scaling (14), the global relations (10)-(12) read

$0=\int e^{\mathbf{i k} \cdot \mathbf{r}}\left[\mu k \sinh \mu k(1+\epsilon \eta) \eta_{t}-\mathbf{i k} \cdot \nabla \Phi^{-} \cosh \mu k(1+\epsilon \eta)\right] d \mathbf{r}$

$$
+\int e^{i \mathbf{k} \cdot \mathbf{r}}\left[-\epsilon \mu k \sinh \left(\epsilon^{2} \mu k b\right) b_{t}+\mathbf{i k} \cdot \nabla \Phi^{b} \cosh \left(\epsilon^{2} \mu k b\right)\right] d \mathbf{r},
$$

$$
\begin{aligned}
0= & \int e^{\mathrm{i} \mathbf{k} \cdot \mathbf{r}}\left[\cosh \mu k(1+\epsilon \eta) \eta_{t}-\mathrm{i} \mathbf{k} \cdot \nabla \Phi^{-} \frac{\sinh \mu k(1+\epsilon \eta)}{\mu k}\right] d \mathbf{r} \\
& +\int e^{\mathbf{i k} \cdot \mathbf{r}}\left[-\epsilon \cosh \left(\epsilon^{2} \mu k b\right) b_{t}+\mathrm{ik} \cdot \nabla \Phi^{b} \frac{\sinh \left(\epsilon^{2} \mu k b\right)}{\mu k}\right] d \mathbf{r},
\end{aligned}
$$

and

$0=\int e^{\mathbf{i k} \cdot \mathbf{r}}\left[\cosh \mu k(\tau-\epsilon \eta) \eta_{t}+\mathrm{ik} \cdot \nabla \Phi^{+} \frac{\sinh \mu k(\tau-\epsilon \eta)}{\mu k}\right] d \mathbf{r}$.

The dynamic boundary condition (13) becomes

$\left(\Phi^{-}-\sigma \Phi^{+}\right)_{t}+\eta+\frac{\epsilon}{2}\left|\nabla \Phi^{-}\right|^{2}-\frac{\epsilon \sigma}{2}\left|\nabla \Phi^{+}\right|^{2}-\frac{\epsilon \mu^{2}}{2} \frac{\left(\eta_{t}+\epsilon \nabla \eta \cdot \nabla \Phi^{-}\right)^{2}}{1+\epsilon^{2} \mu^{2}|\nabla \eta|^{2}}$

$+\frac{\epsilon \mu^{2} \sigma}{2} \frac{\left(\eta_{t}+\epsilon \nabla \eta \cdot \nabla \Phi^{+}\right)^{2}}{1+\epsilon^{2} \mu^{2}|\nabla \eta|^{2}}=0$.

Creating Maclaurin series expansions for the global relations (16)-(17) and retaining terms valid up to $\mathcal{O}\left(\epsilon, \mu^{2}\right)$ yield

$$
\begin{array}{r}
\int e^{\mathbf{i k} \cdot \mathbf{r}}\left[\mu^{2} k^{2} \eta_{t}-\mathbf{i k} \cdot \nabla \boldsymbol{\Phi}^{-}\left(1+\frac{1}{2} \mu^{2} k^{2}\right)+\mathrm{i} \mathbf{k} \cdot \nabla \boldsymbol{\Phi}^{b}\right] d \mathbf{r}=0, \\
\int e^{\mathrm{ik} \cdot \mathbf{r}}\left[\left(1+\frac{1}{2} \mu^{2} k^{2}\right) \eta_{t}-\mathbf{i k} \cdot \nabla \boldsymbol{\Phi}^{-}\left(1+\epsilon \eta+\frac{1}{6} \mu^{2} k^{2}\right)-\epsilon b_{t}\right] d \mathbf{r}=0, \\
\int e^{\mathrm{ik} \cdot \mathbf{r}}\left[\left(1+\frac{1}{2} \mu^{2} k^{2} \tau^{2}\right) \eta_{t}+\mathbf{i k} \cdot \nabla \boldsymbol{\Phi}^{+}\left(\tau-\epsilon \eta+\frac{1}{6} \mu^{2} k^{2} \tau^{3}\right)\right] d \mathbf{r}=0 .
\end{array}
$$

In the same vein, one can obtain the approximation of the dynamic boundary condition (18)

$$
\left(\Phi^{-}-\sigma \Phi^{+}\right)_{t}+\eta+\frac{\epsilon}{2}\left|\nabla \Phi^{-}\right|^{2}-\frac{\epsilon \sigma}{2}\left|\nabla \Phi^{+}\right|^{2}=0 .
$$

Upon noting ik $\sim-\nabla$ and $k^{2} \sim-\Delta$, the inverse Fourier transforms of (19)-(21) give

$$
\begin{array}{r}
\Phi^{-}-\Phi^{b}-\mu^{2}\left(\eta_{t}+\frac{1}{2} \Delta \Phi^{-}\right)=0, \\
\left(1-\frac{\mu^{2}}{2} \Delta\right) \eta_{t}+\left(\Delta-\frac{\mu^{2}}{6} \Delta^{2}\right) \Phi^{-}+\epsilon \nabla \cdot\left(\eta \nabla \Phi^{-}\right)-\epsilon b_{t}=0, \\
\left(1-\frac{\mu^{2} \tau^{2}}{2} \Delta\right) \eta_{t}-\left(\tau \Delta-\frac{\mu^{2} \tau^{3}}{6} \Delta^{2}\right) \Phi^{+}+\epsilon \nabla \cdot\left(\eta \nabla \Phi^{+}\right)=0 .
\end{array}
$$

Eq. (23) is used to decouple $\Phi^{b}$ from the system, while (24) and (25) can be respectively rewritten as

$$
\begin{gathered}
\eta_{t}+\Delta \Phi^{-}+\frac{\mu^{2}}{3} \Delta^{2} \Phi^{-}+\epsilon \nabla \cdot\left(\eta \nabla \Phi^{-}\right)-\epsilon b_{t}=0, \\
\eta_{t}-\tau \Delta \Phi^{+}-\frac{\mu^{2} \tau^{3}}{3} \Delta^{2} \Phi^{+}+\epsilon \nabla \cdot\left(\eta \nabla \Phi^{+}\right)=0,
\end{gathered}
$$

based on equivalent replacement. It is easy to deduce $\Phi^{-}=-\tau \Phi^{+}+$ $\mathcal{O}\left(\epsilon, \mu^{2}\right)$ from (26) and (27). If we define $\xi=\Phi^{-}-\sigma \Phi^{+}$, then

$\Phi^{+}=-\frac{1}{\tau+\sigma} \xi+\mathcal{O}\left(\epsilon, \mu^{2}\right), \quad \Phi^{-}=\frac{\tau}{\tau+\sigma} \xi+\mathcal{O}\left(\epsilon, \mu^{2}\right)$.

Taking the derivative of Eq. (22) with respect to $t$ and then replacing $\eta_{t}$ with (27), one obtains

$\xi_{t t}+\left[\tau \Delta \Phi^{+}+\frac{\mu^{2} \tau^{3}}{3} \Delta^{2} \Phi^{+}-\epsilon \nabla \cdot\left(\eta \nabla \Phi^{+}\right)\right]+\frac{\epsilon}{2}\left|\nabla \Phi^{-}\right|_{t}^{2}-\frac{\epsilon \sigma}{2}\left|\nabla \Phi^{+}\right|_{t}^{2}=0$.

In Eq. (29), $\Phi^{ \pm}$associated with $\epsilon$ and $\mu$ can be replaced by $\xi$ directly using (28). It then suffices to deal with $\Delta \Phi^{+}$. Subtracting (26) from (27) yields

$\Delta \Phi^{+}=-\frac{1}{\tau+\sigma}\left\{\Delta \xi+\frac{\mu^{2}}{3} \Delta^{2}\left(\Phi^{-}+\tau^{3} \Phi^{+}\right)+\epsilon \nabla \cdot\left[\eta \nabla\left(\Phi^{-}-\Phi^{+}\right)\right]-\epsilon b_{t}\right\}$

To derive the governing equation, we substitute (30) into (29), replace $\eta$ with $-\xi_{t}$, cancel $\Phi^{ \pm}$using (28), and retain terms valid up to $\mathcal{O}\left(\epsilon, \mu^{2}\right)$. 
A tedious but straightforward calculation results in a time evolution equation for $\xi$ :

$\xi_{t t}-\frac{\tau}{\tau+\sigma} \Delta \xi-\frac{\mu^{2} \tau^{2}(1+\tau \sigma)}{3(\tau+\sigma)^{2}} \Delta^{2} \xi+\frac{\epsilon \tau}{\tau+\sigma} b_{t}+\frac{\epsilon\left(\tau^{2}-\sigma\right)}{(\tau+\sigma)^{2}}\left[|\nabla \xi|_{t}^{2}+\xi_{t} \Delta \xi\right]=0$.

When $\sigma=0$ and $b=0$, that is no stratification and bottom topography, Eq. (31) reduces to the so-called Benney-Luke equation (see Benney and Luke, 1964 for details). Thus Eq. (31) is named the modified Benney-Luke equation. To study solitary waves, we usually assume $\epsilon=\mathcal{O}\left(\mu^{2}\right)$ to balance dispersion and nonlinearity. Returning back to physical space, in dimensional variables the corresponding equation is

$$
\begin{aligned}
\xi_{t t}- & \frac{g h^{+}(1-\sigma)}{\tau+\sigma} \Delta \xi-\frac{g\left(h^{+}\right)^{2}\left(h^{-}+\sigma h^{+}\right)(1-\sigma)}{3(\tau+\sigma)^{2}} \Delta^{2} \xi \\
+ & \frac{g \tau(1-\sigma)}{\tau+\sigma} b_{t}+\frac{\tau^{2}-\sigma}{(\tau+\sigma)^{2}}\left[|\nabla \xi|_{t}^{2}+\xi_{t} \Delta \xi\right]=0 .
\end{aligned}
$$

It is well known that the linear dispersion relation for the two-layer interfacial wave problem takes the form

$\omega^{2}=\frac{g k\left(\rho^{-}-\rho^{+}\right) \tanh \left(h^{-} k\right) \tanh \left(h^{+} k\right)}{\rho^{-} \tanh \left(h^{+} k\right)+\rho^{+} \tanh \left(h^{-} k\right)}$.

For long waves, we can expand the dispersion relation at $k=0$ using the Taylor series:

$\omega^{2}=\frac{g h^{+}(1-\sigma) k^{2}}{\tau+\sigma}-\frac{g\left(h^{+}\right)^{2}(1-\sigma)\left(h^{-}+\sigma h^{+}\right) k^{4}}{3(\tau+\sigma)^{2}}+\cdots$,

which verifies the linear part of the resulting equation.

Note that it seems quite difficult to find analytical solutions to the modified Benney-Luke Equation (31) or (32), and hence we will resort to numerical methods. Nonetheless, the dispersion relation (34) indicates that the proposed model is linearly ill-posed since the frequency $\omega$ becomes purely imaginary for large wavenumbers $k$, therefore, for long waves, an equivalent replacement $\xi_{t t}=c^{2} \Delta \xi$ (where $c$ is the linear wave phase speed as given below) as pointed out by Milewski and Tabak (1999) is used. Then Eq. (32) can be rewritten as

$\xi_{t t}-c^{2} \Delta \xi-\alpha \Delta \xi_{t t}+\beta b_{t}+\gamma\left[|\nabla \xi|_{t}^{2}+\xi_{t} \Delta \xi\right]=0$

with the coefficients

$$
\begin{array}{r}
c^{2}=\frac{g h^{+}(1-\sigma)}{\tau+\sigma}, \quad \alpha=\frac{h^{+}\left(h^{-}+\sigma h^{+}\right)}{3(\tau+\sigma)}, \quad \beta=\frac{g \tau(1-\sigma)}{\tau+\sigma}, \\
\gamma=\frac{\tau^{2}-\sigma}{(\tau+\sigma)^{2}}, \quad \tau=\frac{h^{+}}{h^{-}}, \quad \sigma=\frac{\rho^{+}}{\rho^{-}}<1 .
\end{array}
$$

Note that in the realistic ocean, $\sigma \sim 1$, and $\beta$ is usually small. In dimensional variables, the leading-order approximation of the interface $\eta(x, y, t)$ can be expressed as

$\eta=-\frac{\xi_{t}}{g(1-\sigma)}$.

It is clear that the modified Benney-Luke equation without bottom topography admits solitary-wave solutions propagating in any direction given by

$\xi_{t}=A(k) \operatorname{sech}^{2}(\mathbf{k} \cdot \mathbf{r}-v k t), \quad \xi=-\frac{A}{v k} \tanh (\mathbf{k} \cdot \mathbf{r}-v k t)+\mathbf{P} \cdot \mathbf{r}$,

where the wavevector $\mathbf{k}=\left(k_{x}, k_{y}\right)$ is in the $\mathbf{r}=(x, y)$ direction, with the modulus of $k=\left(k_{x}^{2}+k_{y}^{2}\right)^{1 / 2}$. When numerical experiments are carried out in a periodic box, the constant $\mathbf{P}$ is chosen to keep $\xi$ periodic. The amplitude $A(k)$ and nonlinear wave speed $v(k)$ are in the form of

$A(k)=-\frac{4 \alpha v^{2} k^{2}}{\gamma}, \quad v^{2}=\frac{c^{2}}{1-4 \alpha k^{2}}$.

It is worth pointing out that the modified Benney-Luke equation lays its foundation on the assumption of weak nonlinearity, thus much care should be given when it is used to delineate large-amplitude waves, albeit its formal range of validity still needs to be further determined. Compared with the widely used unidirectional two-dimensional KdV-type theory, usually called the KP equation (Kadomtsev and Petviashvili, 1970; Grimshaw and Melville, 1989; Yuan et al., 2018a), the present model is bidirectional and isotropic. Indeed, the KP equation is a limiting case of the modified Benney-Luke equation in quasitwo-dimensional situations. We can make further simplification by assuming that waves are unidirectionally propagating and the variations in the transverse direction $y$ are much weaker than those in the wave propagation direction $x$. Let

$X=x-\tilde{c} t, \quad T=\epsilon t, \quad Y=\mu y, \quad \tilde{c}=[\tau /(\tau+\sigma)]^{1 / 2}$,

thus

$\partial_{t}=-\tilde{c} \partial_{X}+\epsilon \partial_{T}, \quad \partial_{x}=\partial_{X}, \quad \partial_{y}=\mu \partial_{Y}$.

Recalling that the relation $\epsilon=\mathcal{O}\left(\mu^{2}\right)$, Eq. (31) reduces to

$2 \xi_{X T}+\frac{\mu^{2}}{\epsilon} \frac{\tau \tilde{c}(1+\tau \sigma)}{3(\tau+\sigma)} \xi_{X X X X}+\frac{3\left(\tau^{2}-\sigma\right)}{(\tau+\sigma)^{2}} \xi_{X} \xi_{X X}+\tilde{c}^{2} b_{X}+\frac{\mu^{2} \tilde{c}}{\epsilon} \xi_{Y Y}=0$,

which is asymptotically valid up to $\mathcal{O}\left(\epsilon, \mu^{2}\right)$. Taking derivative with respect to $X$ and defining $Y=\xi_{X}$ yield

$\left[2 Y_{T}+\frac{\mu^{2}}{\epsilon} \frac{\tau \tilde{c}(1+\tau \sigma)}{3(\tau+\sigma)} Y_{X X X}+\frac{3\left(\tau^{2}-\sigma\right)}{(\tau+\sigma)^{2}} Y Y_{X}+\tilde{c}^{2} b_{X}\right]_{X}+\frac{\mu^{2} \tilde{c}}{\epsilon} Y_{Y Y}=0$,

which is the KP equation. This degeneration from the modified BenneyLuke equation to the KP equation implies the former has a wider scope of applicability than the latter one, especially when the variations in the transverse direction are comparable with those in the direction of wave propagation.

\subsection{Numerical method}

To obtain accurate numerical results of the modified Benney-Luke equation, the numerical techniques proposed by Milewski and Tabak (1999) are implemented. Here we just briefly described the procedure. Note that Eq. (35) can be rewritten as

$\left[\frac{\partial^{2}}{\partial t^{2}}+M^{2}\right] \xi=G\left(\xi, \xi_{t}, \nabla \xi\right)$

where the operators $M$ and $G$ are, respectively,

$M^{2}=-\frac{c^{2} \Delta}{I-\alpha \Delta}, \quad G=-\frac{1}{I-\alpha \Delta}\left\{\beta b_{t}+\gamma\left[|\nabla \xi|_{t}^{2}+\xi_{t} \Delta \xi\right]\right\}$.

Next, introducing an intermediate variable $\theta$,

$\theta=\left(\frac{\partial}{\partial t}+i M\right) \xi$

as a result, Eq. (44) reduces to a first-order one,

$\left(\frac{\partial}{\partial t}-i M\right) \theta=G\left(\xi, \xi_{t}, \nabla \xi\right)$.

Taking the two-dimensional Fourier transform of (47) with respect to the spatial variables yields

$\left(\frac{\partial}{\partial t}-i \widehat{M}\right) \hat{\theta}=\mathfrak{F}\left[G\left(\xi, \xi_{t}, \nabla \xi\right)\right]$,

where $\mathfrak{F}$ represents the Fourier transform. Then multiplying (48) by an integrating factor $e^{-i \widehat{M} t}$, one obtains

$\frac{d \widehat{\Theta}}{d t}=e^{-i \widehat{M} t} \mathfrak{F}\left[G\left(\xi, \xi_{t}, \nabla \xi\right)\right], \quad \widehat{\Theta}=e^{-i \widehat{M} t} \widehat{\theta}$.

It remains to relate $\widehat{\Theta}$ or $\hat{\theta}$ with $\xi$ and $\xi_{t}$. Taking the Fourier transform of (46) in the spatial domain and after a simple manipulation, we get

$\widehat{\xi}(k, t)=\frac{\widehat{\theta}(k, t)-\widehat{\theta}^{*}(-k, t)}{i[\widehat{M}(k)+\widehat{M}(-k)]}, \quad \widehat{\xi}_{t}(k, t)=\frac{\widehat{\theta}(k, t)+\widehat{\theta}^{*}(-k, t)}{2}$,

where the superscript asterisk indicates the complex conjugate. In the temporal domain, a classical fourth-order Runge-Kutta method is used. 


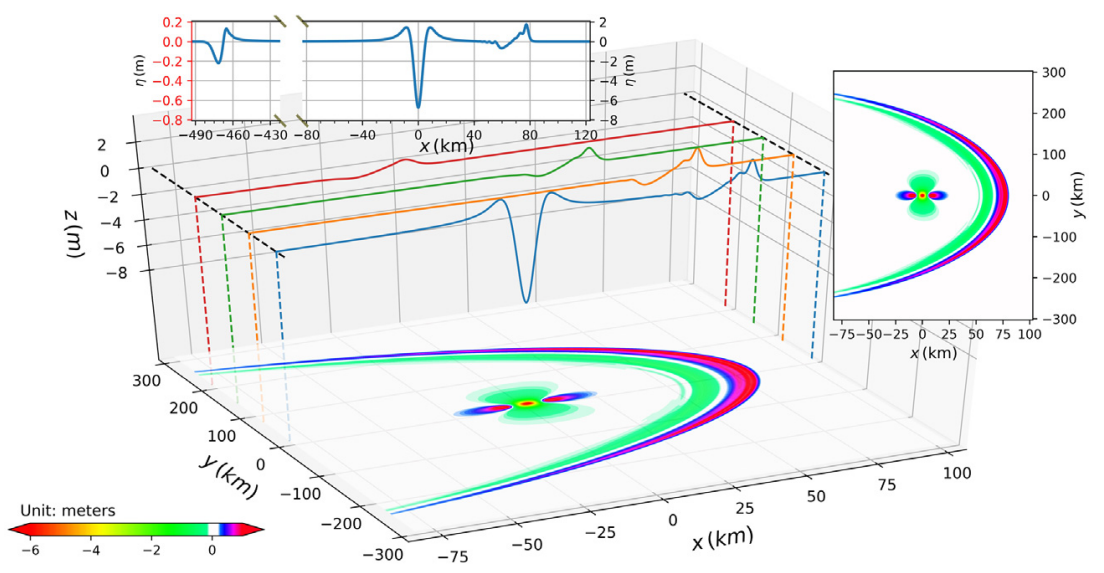

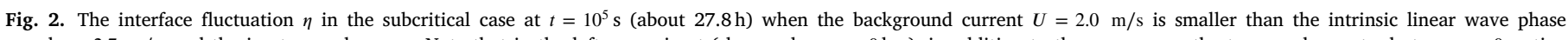

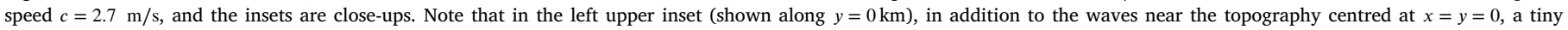
counterpart in the negative $x$-axis is illustrated in different scales (labelled in the colour of red).

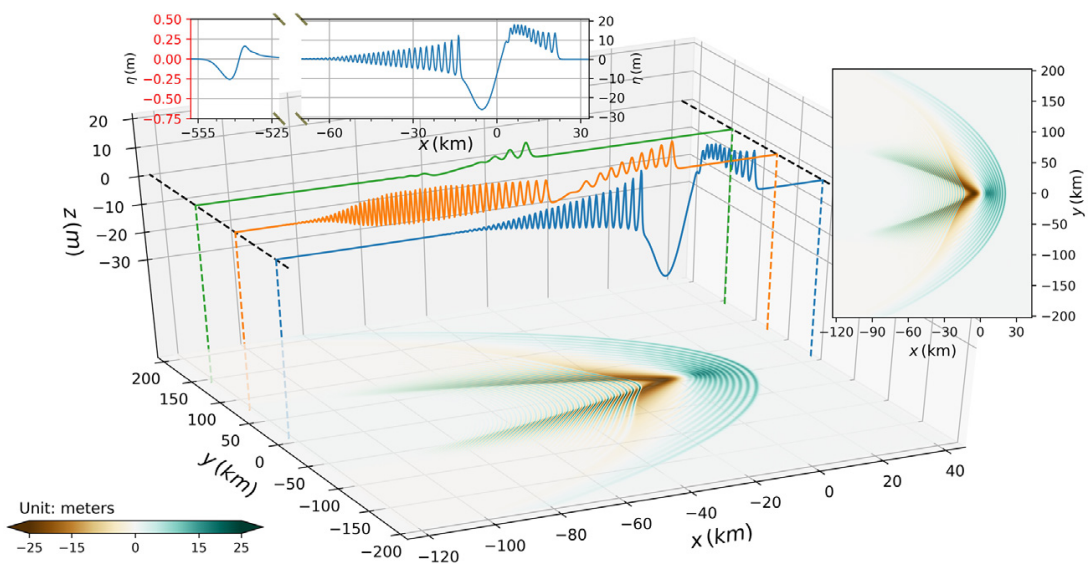

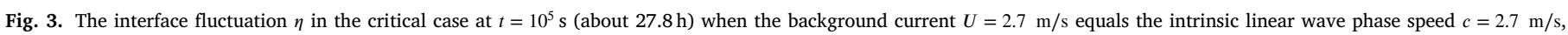
and the insets are close-ups. The layout is the same as in Fig. 2.

\section{Generation of internal waves}

In this section, using the modified Benney-Luke equation, waves generated by a background current passing over topography are numerically simulated. To better illustrate the results, the scales of simulations are chosen as in realistic ocean conditions. Here the spatial ranges are $x \times y=[-500: 500] \times[-500: 500] \mathrm{km}^{2}$, discretized by 5000 (or 3000 ) and 3000 grids respectively, and the time step is set to be $10 \mathrm{~s}$. The stratification is simplified to two layers, with thicknesses of 100 and $300 \mathrm{~m}$, densities of 1020 and $1030.2 \mathrm{~kg} / \mathrm{m}^{3}$ for the upper and lower layers. A Gaussian topography, delineated by $b=b_{0} \exp \left[-\left(x / x_{w}\right)^{2}-\left(y / y_{w}\right)^{2}\right]$, where the height $b_{0}=50 \mathrm{~m}$ and the characteristic width $x_{w}=y_{w}=$ $5000 \mathrm{~m}$, is taken into consideration (see the schematic diagram in Fig. 1). One pending problem is how to incorporate background currents into the system, since no terms representing background currents arise explicitly in the derivation (see Section 2). To circumvent this difficulty, note that the topography $b(x, y, t)$ is dependent on time $t$, therefore a background current flowing over the topography amounts to the case in which the bottom topography $b$ moves with the velocity of the background current, but in the opposite direction, a technique similar to Gerkema and Zimmerman (1995). Nevertheless, to understand the underlying physics clearly, the moving topography is shifted to the origin $x=y=0$ for the results exhibited in Figs. 2-8.

\subsection{Constant background current}

In this model set-up, the intrinsic linear wave phase speed $c=$ $2.7 \mathrm{~m} / \mathrm{s}$ as given in Eq. (36). We consider three cases with constant background current $U=2,2.7$ and $3.2 \mathrm{~m} / \mathrm{s}$, corresponding to the subcritical $U<c$, critical $U \approx c$ and supercritical $U>c$ scenarios. Since the modified Benney-Luke equation is isotropic, the constant background current $U$ is aligned along the negative $x$-axis.

It is clear that the generation falls into the regime of lee wave mechanism in all three cases (see Figs. 2-5). In the subcritical regime (see Fig. 2), a steady lee wave emanates from the topography and propagates in the positive $x$ direction with a velocity of $c-U=0.7 \mathrm{~m} / \mathrm{s}$, detaching from a forced stationary solitary wave arising over the topography. In addition, a free and small-amplitude $\left(\mathcal{O}\left(10^{-1}\right)\right)$ counterpart emerges in the negative direction $(x<0)$ and propagates with its own intrinsic velocity $c=-2.7 \mathrm{~m} / \mathrm{s}$ superimposed on the background current $U=-2 \mathrm{~m} / \mathrm{s}$, as a result, $c+U=-4.7 \mathrm{~m} / \mathrm{s}$. Meanwhile, waves also disperse in the transverse direction, which leads to the occurrence of the "arc". In contrast, when the scenario is in the critical regime, apart from a similar free wave in the negative $x$ direction, waves are confined near the topography, both downstream and upstream, and the superimposed velocity tends to zero (Fig. 3). The waveforms are characterized by a modulated cnoidal-like wavetrain downstream, akin 

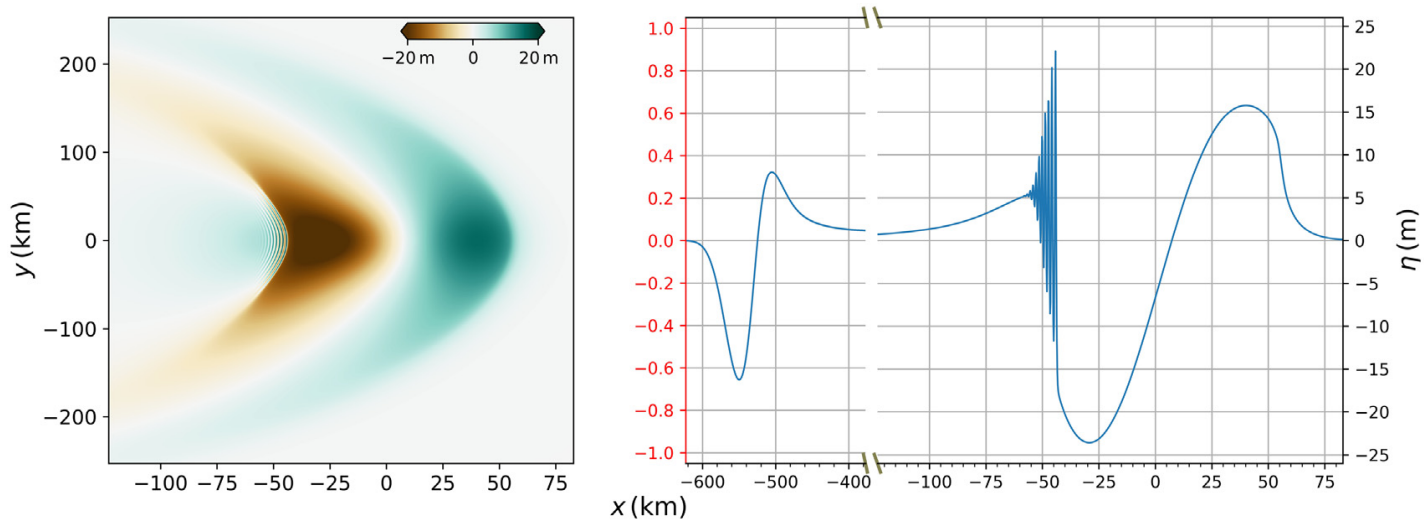

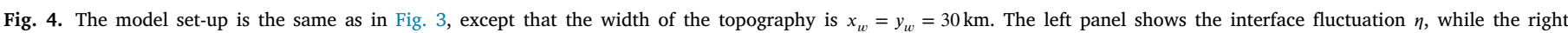
panel along $y=0$.

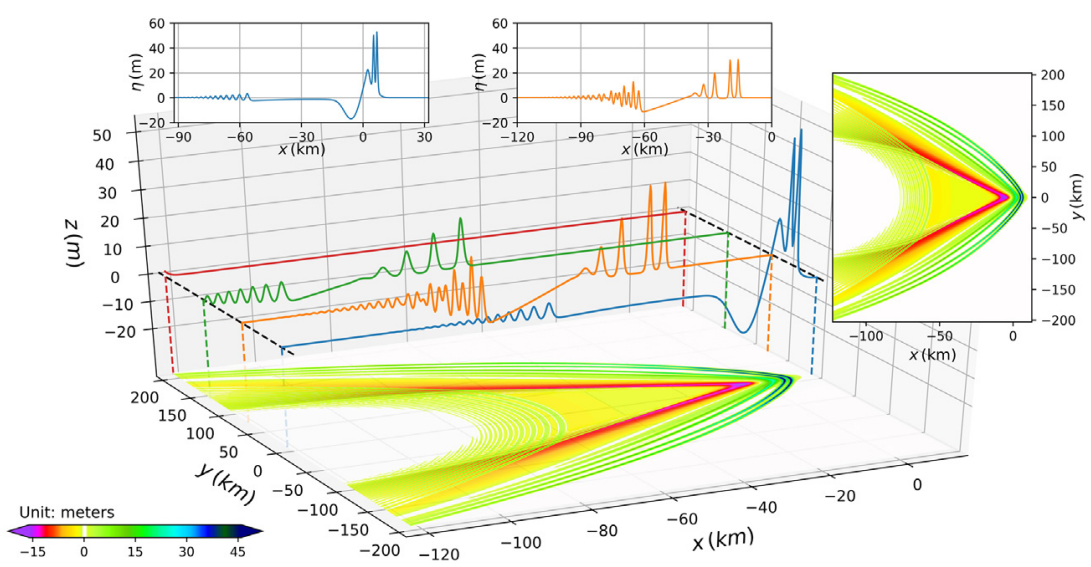

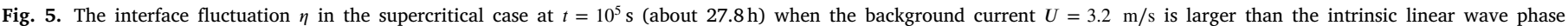

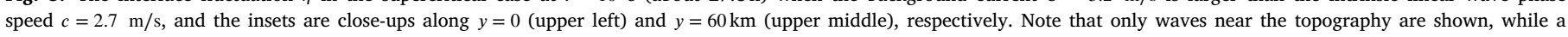
small-amplitude counterpart propagating in the negative $x$-axis is not illustrated.

to the one-dimensional case (see, for instance, Grimshaw and Smyth (1986) and Grimshaw and Helfrich (2018). In the one-dimensional model, waves upstream are a train of solitary waves of nearly uniform amplitude, however, in the result shown in Fig. 3, the upstream waves are instead in the form of an undular bore riding on the elevation level. The reason for this can be ascribed to the dispersive effects in the $y$ direction, and indeed, the dispersive effects lead to a peculiar wave pattern, whose appearance is quite similar to the well-known Kelvin wake excited by a ship moving over the water surface, albeit here the half-angle is $40^{\circ}$ approximately, which is much larger than the well-known $19.47^{\circ}$ for surface waves in deep water (Dias, 2014). The results illustrated in Fig. 4 show that this angle increases with the width of the topography (cf. Fig. 3). In the supercritical case (Fig. 5), the most pronounced feature is the solitary waves over the topography. In the one-dimensional model, such a solitary wave is stationary, while in the present model, the dispersive effects in the transverse direction destroy the steady waveform, and it disintegrates into a train of nonlinear waves. On the other hand, two additional wavetrains propagate away from the topography both in the negative $x$ direction, with the respective velocities of $U+c=-5.9 \mathrm{~m} / \mathrm{s}$ and $-0.5 \mathrm{~m} / \mathrm{s}$. We remark that all of these findings are applicable only to narrow topographies, whereas the scenarios are quite different for wide topographies (see, for example, Fig. 4).

\subsection{Barotropic tides}

In the ocean, the interaction between barotropic tides and topographic features is considered as the primary generation mechanism of internal waves. Here we consider two categories of tidal currents with a period of $12 \mathrm{~h}$ : one is only aligned with the $x$-axis, but with two types of maximum strength, $U_{m}=1<c=2.7 \mathrm{~m} / \mathrm{s}$ and $U_{m}=3.6>c=2.7 \mathrm{~m} / \mathrm{s}$, while for the other case, velocity components exist in both $x$ and $y$ directions, with the maximum strength $U=V=3.6 \mathrm{~m} / \mathrm{s}$, and $U$ lags behind $V$ by a phase of $\pi / 2$ in the $y$ direction.

When the maximum strength of the tidal current is $1 \mathrm{~m} / \mathrm{s}$, the flow is always subcritical. In addition to successive waves appearing over the topography, there exist waves propagating away from the bump in the upstream and downstream directions as the tidal current reverses direction. As shown in Fig. 6, the combined effects of nonlinearity and dispersion in both the $x$ and $y$ directions also affect the wave evolution and modulate the sinusoidal-like waveform to embody the traits which are not accommodated in the linear theory. This feature is more prominent when the forcing is larger. Fig. 7 illustrates the case when the tidal current passes through the critical regime four times in one period. Starting from zero at $t=0$, the barotropic tide increases its strength in the positive $x$ direction, and as in the subcritical case, waves first appear over the topography, together with freely propagating 


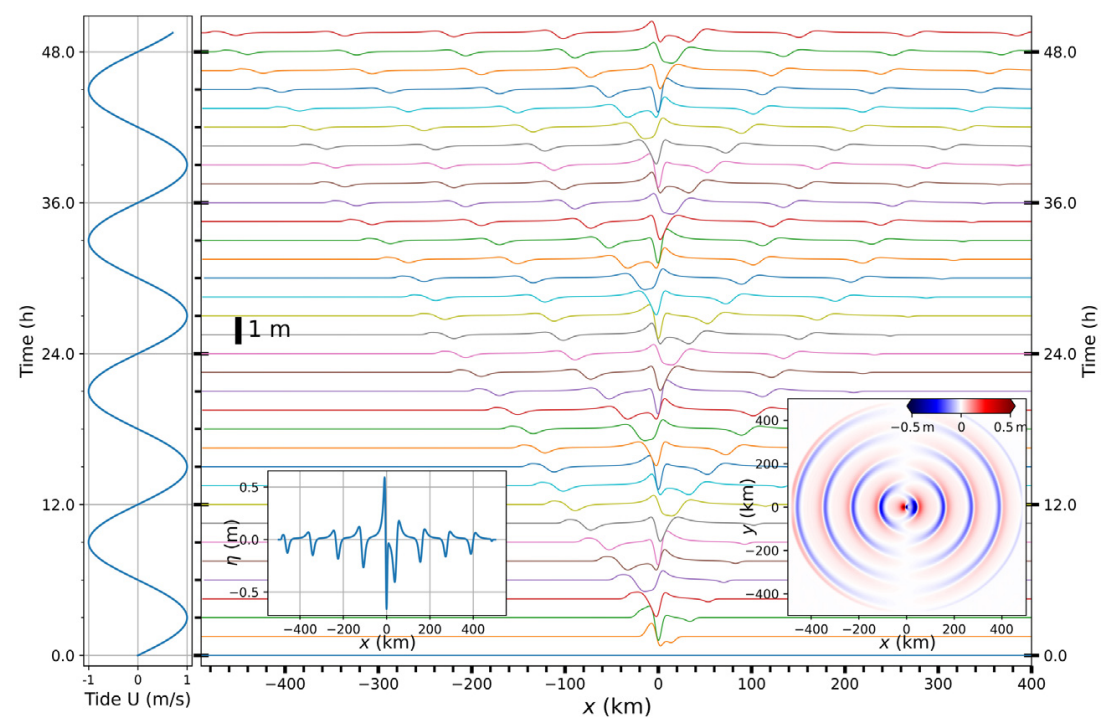

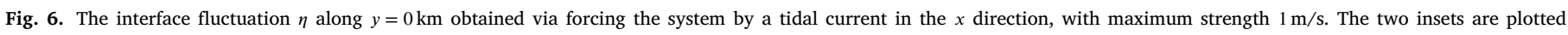
at time $t=50 \mathrm{~h}$, and the left one is the close-up of $\eta$ at the central line $y=0$.

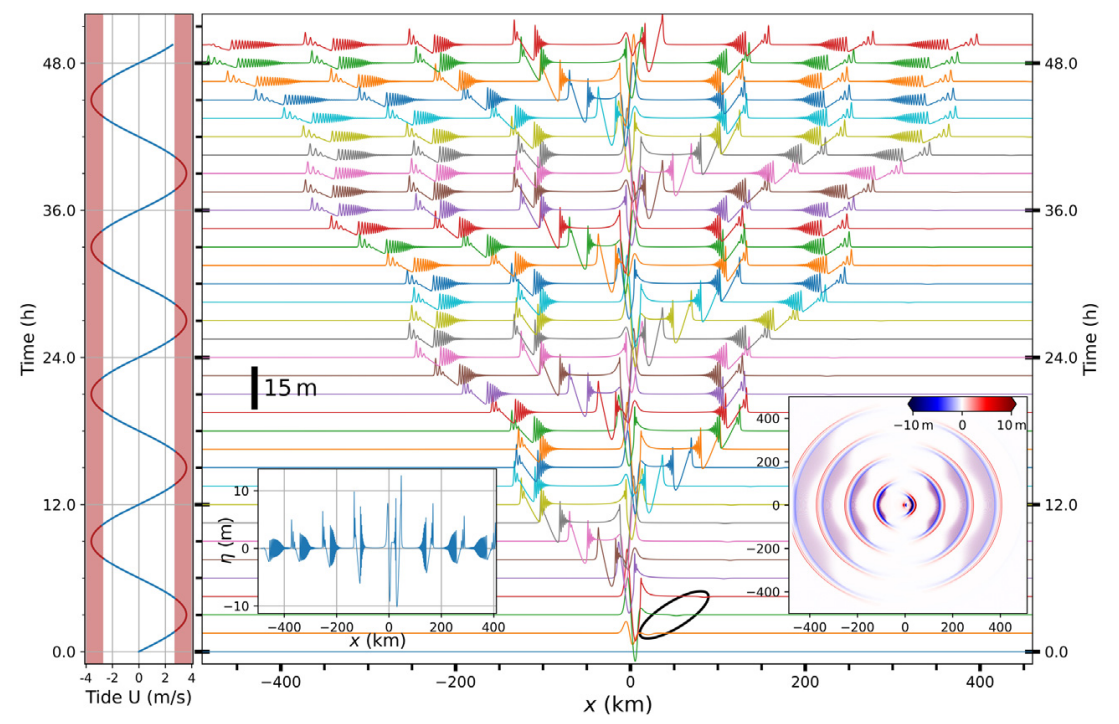

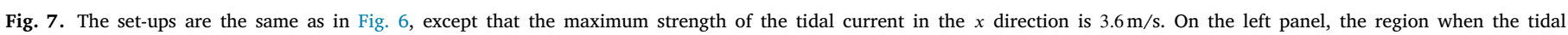
current is larger than the intrinsic linear wave phase speed is accentuated in shadow.

small-amplitude counterparts (the dark ellipse in Fig. 7). At $t=1.6 \mathrm{~h}$, the scenario passes through the critical speed and turns into the supercritical regime. As the tidal current increases, the nascent nonlinear wavetrains grow and deform until they detach from the topography when the tidal current changes direction at $t=6 \mathrm{~h}$. In the next half period $t=6-12 \mathrm{~h}$, the emitting waves feature a waveform composed of leading solitary wavetrains and the subsequent undular bores and propagate with the velocity of $U(t)+c$, which is always negative. At the same time, on the opposite side of the topography, a similar generation process repeats, and again these waves are released when the tidal current changes its direction at $t=12 \mathrm{~h}$. In the second period, the tidal current becomes positive again and waves propagating in the negative $x$ direction experience decelerations. When the tidal current enters the supercritical regime, these waves even propagate backward towards the topography slightly. This process cycles at every period and results in the intermittent generation of nonlinear wavetrains. When it is forced by a two-dimensional tidal current which are $3.6 \mathrm{~m} / \mathrm{s}$ in both directions, but out of phase by $\pi / 2$, the remarkable feature is a spiral wave pattern emanating from the bump in the horizontal plane (Fig. 8), which verifies that the model is isotropic.

\section{Discussion and conclusion}

While oceanic nonlinear internal waves are essentially threedimensional, most of the theories currently used are horizontally onedimensional, which, to some extent, hinders the understanding of these waves. Compared with much effort has been devoted to the study in three dimensions via numerical simulations (Vlasenko and Stashchuk, 2007; Buijsman et al., 2014 amongst many others) and laboratory experiments (Mercier et al., 2013; Wang et al., 2017 for instance), 


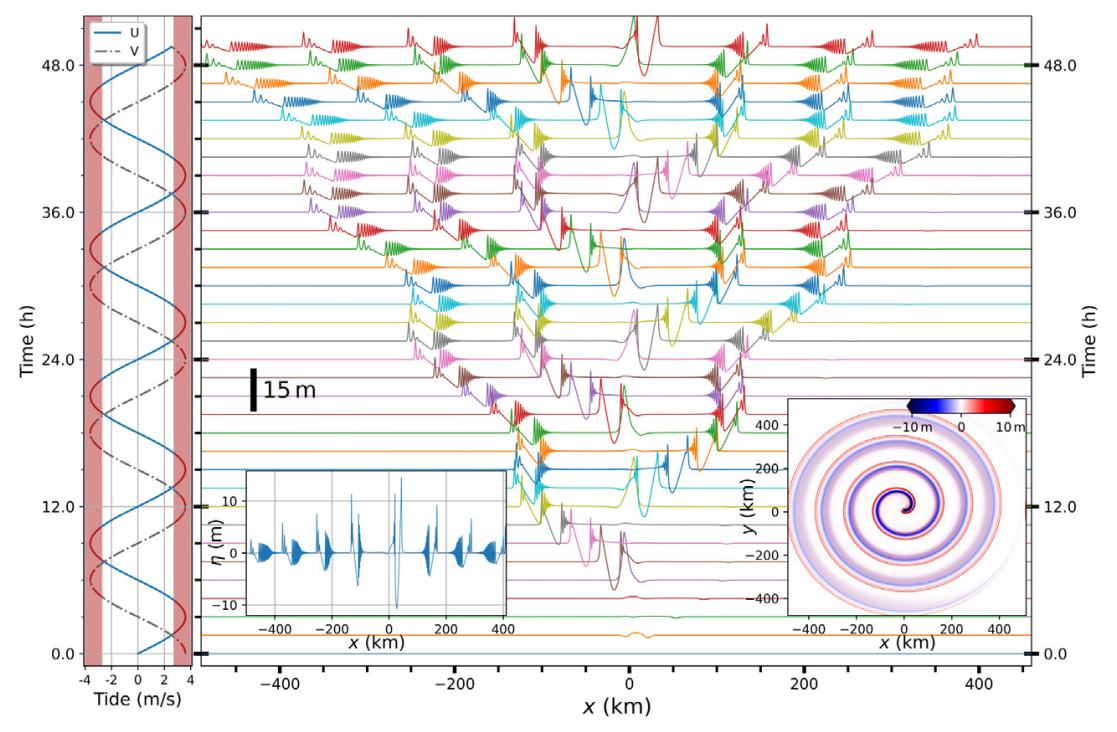

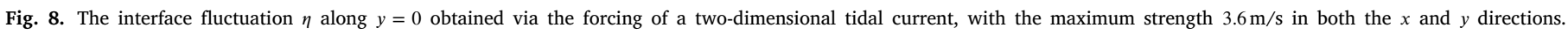
There is a phase lag of $\pi / 2$ in the $y$ direction. The layout of two insets is the same as in Fig. 6 .

theoretical explorations are reported rarely. The few studies we are aware of, for example Lee and Grimshaw (1990), Cai and Xie (2010), Chen et al. (2011), Yuan et al. (2018a) and Yuan et al. (2018b), are all based on the KP equation, which plausibly inherits the wide range of validity held by its parent, the well-known and widely used KdV equation. Especially, Yuan et al. (2018a) illustrated satisfactory comparisons between the KP equation and the primitive Navier-Stokes equations. Notwithstanding all these advantages and the accumulation of relevant mathematical analyses, the KP equation has two limitations, one is its unidirectional nature, which makes it unable to investigate wave reflection or deflection, and the other one is anisotropy: it is not adequate for phenomena with large angles, and indeed, Ablowitz and Curtis (2013) exhibited a discrepancy between the KP equation and the Benney-Luke equation for free-surface waves.

To theoretically investigate three-dimensional internal waves and overcome the shortcomings of the KP equation, we derive an isotropic bidirectional model, the modified Benney-Luke equation. Adopting the common assumptions of incompressibility, non-rotation, nondissipation and long waves, we start from two Laplace's equations in a two-layer fluid system, and then carry out asymptotic analyses balancing nonlinearity and dispersion via choosing $\epsilon=\mathcal{O}\left(\mu^{2}\right)$. The resulting modified Benney-Luke equation can be easily reduced to the classic Benney-Luke equation for surface waves, and moreover, it can also be simplified to the KP equation under the correct assumptions. Nonetheless, the trade-off is that the structure of the resulting equation is not as succinct as the KdV-type equations, and it is difficult to find analytical solutions to the proposed equation, thus a specific numerical scheme is suggested in Section 2.3. Based on this model, the generation processes of nonlinear internal waves excited by constant background currents and barotropic tides flowing over topography are examined, demonstrating the reliability and effectiveness of the model.

Finally, it is necessary to adumbrate potential shortcomings. In some cases the small terms at the order of $o\left(\epsilon, \mu^{2}\right)$ that have been ignored in the present derivation may be important necessitating the inclusion of higher-order terms. To determine conditions under which they should be included, we need to conduct comparisons with in-situ observations, laboratory experiments or three-dimensional numerical simulations based on the primitive Navier-Stokes equations. These comparisons are really worthwhile, as they can help to identify the range of validity of the modified Benney-Luke equation. In addition, the present equation stems from the assumption of two fluid layers, which is a good approximation for some oceanic environment, but not applicable for investigating mode- 2 and even higher mode internal waves, therefore extensions to multiple layers and continuous stratification are still waiting to be developed in further studies.

\section{CRediT authorship contribution statement}

C. Yuan: Software, Writing - original draft, Visualization, Data curation. Z. Wang: Conceptualization, Methodology, Writing - review \& editing, Validation. X. Chen: Validation, Resources, Supervision.

\section{Declaration of competing interest}

The authors declare that they have no known competing financial interests or personal relationships that could have appeared to influence the work reported in this paper.

\section{Acknowledgements}

This work was supported by the National Natural Science Foundation of China (Nos. 11911530171, 11772341), the Key Program of National Natural Science Foundation of China (NO. 91958206), the Key Research Program of Frontier Sciences, China, Chinese Academy of Sciences (No. QYZDBSSW-SYS015), and the Strategic Priority Research Program of the Chinese Academy of Sciences (No. XDB22040203). The authors wish to thank Professor Roger Grimshaw and Professor Wai Sun Dong for comments on earlier draft versions of this paper. The authors would like to thank anonymous reviewers for helpful suggestions and comments.

\section{References}

Ablowitz, M.J., Curtis, C.W., 2013. Conservation laws and non-decaying solutions for the Benney-Luke equation. Proc. R. Soc. A 469 (2152), 20120690.

Ablowitz, M.J., Fokas, A.S., Musslimani, Z.H., 2006. On a new non-local formulation of water waves. J. Fluid Mech. 562, 313-343.

Benjamin, T.B., 1966. Internal waves of finite amplitude and permanent form. J. Fluid Mech. 25 (2), 241-270.

Benney, D.J., 1966. Long non-linear waves in fluid flows. Stud. Appl. Math. 45 (1-4), 52-63. 
Benney, D.J., Luke, J.C., 1964. On the interactions of permanent waves of finite amplitude. J. Math. Phys. 43 (1-4), 309-313.

Buijsman, M.C., Klymak, J.M., Legg, S., Alford, M.H., Farmer, D., MacKinnon, J.A., Nash, J.D., Park, J.-H., Pickering, A., Simmons, H., 2014. Three-dimensional double-ridge internal tide resonance in Luzon Strait. J. Phys. Oceanogr. 44 (3), 850-869.

Cai, S., Xie, J., 2010. A propagation model for the internal solitary waves in the northern South China Sea. J. Geophys. Res.: Oceans 115 (C12074).

Chen, G.-Y., Liu, C.-T., Wang, Y.-H., Hsu, M.-K., 2011. Interaction and generation of long-crested internal solitary waves in the South China Sea. J. Geophys. Res.: Oceans 116 (C6), C06013.

Choi, W., Camassa, R., 1999. Fully nonlinear internal waves in a two-fluid system. J. Fluid Mech. 396, 1-36.

Curtis, C.W., Shen, S.S.P., 2015. Three-dimensional surface water waves governed by the forced Benney-Luke equation. Stud. Appl. Math. 135 (4), 447-465.

Dias, F., 2014. Ship waves and kelvin. J. Fluid Mech. 746, 1-4.

Djordjevic, V.D., Redekopp, L.G., 1978. The fission and disintegration of internal solitary waves moving over two-dimensional topography. J. Phys. Oceanogr. 8 (6), 1016-1024.

Gerkema, T., Zimmerman, J.T.F., 1995. Generation of nonlinear internal tides and solitary waves. J. Phys. Oceanogr. 25 (6), 1081-1094.

Grimshaw, R., 1981. Evolution equations for long, nonlinear internal waves in stratified shear flows. Stud. Appl. Math. 65 (2), 159-188.

Grimshaw, R., 1985. Evolution equations for weakly nonlinear long internal waves in a rotating fluid. Stud. Appl. Math. 73 (1), 1-33.

Grimshaw, R., 2002. Environmental Stratified Flows. Springer.

Grimshaw, R., Helfrich, K.R., 2018. Internal solitary wave generation by tidal flow over topography. J. Fluid Mech. 839, 387-407.

Grimshaw, R., Melville, W., 1989. On the derivation of the modified KadomtsevPetviashvili equation. Stud. Appl. Math. 80 (3), 183-202.

Grimshaw, R., Pelinovsky, E., Poloukhina, O., 2002. Higher-order Korteweg-de Vries models for internal solitary waves in a stratified shear flow with a free surface. Nonlinear Process. Geophys. 9, 221-235.

Grimshaw, R., Pelinovsky, E., Talipova, T., 2003. Damping of large-amplitude solitary waves. Wave Motion 37 (4), 351-364.

Grimshaw, R.H.J., Smyth, N., 1986. Resonant flow of a stratified fluid over topography. J. Fluid Mech. 169, 429-464.

Haut, T.S., Ablowitz, M.J., 2009. A reformulation and applications of interfacial fluids with a free surface. J. Fluid Mech. 631, 375-396.

Helfrich, K.R., Melville, W.K., 2006. Long nonlinear internal waves. Annu. Rev. Fluid Mech. 38, 395-425.

Holloway, P.E., Pelinovsky, E., Talipova, T., Barnes, B., 1997. A nonlinear model of internal tide transformation on the Australian North West Shelf. J. Phys. Oceanogr. 27 (6), 871-896.

Joseph, R.I., 1977. Solitary waves in a finite depth fluid. J. Phys. A: Math. Gen. 10 (12), L225.

Kadomtsev, B.B., Petviashvili, V.I., 1970. On the stability of solitary waves in weakly dispersing media. Sov. Phys. Dokl. 15, 539-541.

Kubota, T., Ko, D.R.S., Dobbs, L.D., 1978. Weakly-nonlinear, long internal gravity waves in stratified fluids of finite depth. J. Hydronaut. 12 (5), 157.

Kunze, E., 2017. Internal-wave-driven mixing: Global geography and budgets. J. Phys. Oceanogr. 47, 1325-1345.
Lamb, K.G., 2003. Shoaling solitary internal waves: on a criterion for the formation of waves with trapped cores. J. Fluid Mech. 478, 81-100.

Lee, C.-Y., Beardsley, R.C., 1974. The generation of long nonlinear internal waves in a weakly stratified shear flow. J. Geophys. Res. 79 (3), 453-462.

Lee, S., Grimshaw, R.H.J., 1990. Upstream advancing waves generated by three-dimensional moving disturbances. Phys. Fluids A 2, 194-201.

Long, R.R., 1955. Some aspects of the flow of stratified fluids. Tellus 7 (3), 341-357.

Mercier, M.J., Gostiaux, L., Helfrich, K., Sommeria, J., Viboud, S., Didelle, H., Ghaemsaidi, S.J., Dauxois, T., Peacock, T., 2013. Large-scale, realistic laboratory modeling of $\mathrm{M}_{2}$ internal tide generation at the Luzon Strait. Geophys. Res. Lett. 40 (21), 5704-5709.

Milewski, P., Tabak, E., 1999. A pseudospectral procedure for the solution of nonlinear wave equations with examples from free-surface flows. SIAM J. Sci. Comput. 21 (3), 1102-1114.

Miyata, M., 1985. An internal solitary wave of large amplitude. La Mer 23 (2), 43-48.

Miyata, M., 1988. Long internal waves of large amplitude. In: Proceedings of the IUTAM Symposium on Nonlinear Water Waves. pp. 399-406.

Ono, H., 1975. Algebraic solitary waves in stratified fluids. J. Phys. Soc. Japan 39 (4), 1082-1091.

Osborne, A.R., Burch, T.L., 1980. Internal solitons in the Andaman Sea. Science 208 (4443), 451-460.

Ostrovsky, L.A., 1978. Nonlinear internal waves in a rotating ocean. Oceanology 18 (2), 119-125.

Ostrovsky, L.A., Grue, J., 2003. Evolution equations for strongly nonlinear internal waves. Phys. Fluids 15 (10), 2934-2948.

Ostrovsky, L.A., Stepanyants, Y.A., 2005. Internal solitons in laboratory experiments: Comparison with theoretical models. Chaos 15 (3), 037111.

Pierini, S., 1989. A model for the Alboran Sea internal solitary waves. J. Phys. Oceanogr. 19 (6), 755-772.

Stastna, M., Lamb, K.G., 2002. Large fully nonlinear internal solitary waves: The effect of background current. Phys. Fluids 14 (9), 2987-2999.

Tung, K.-K., Chan, T.F., Kubota, T., 1982. Large amplitude internal waves of permanent form. Stud. Appl. Math. 66 (1), 1-44.

Vlasenko, V., Stashchuk, N., 2007. Three-dimensional shoaling of large-amplitude internal waves. J. Geophys. Res.: Oceans 112 (C11018).

Wang, C., Pawlowicz, R., 2011. Propagation speeds of strongly nonlinear near-surface internal waves in the Strait of Georgia. J. Geophys. Res.: Oceans 116 (C10).

Wang, J., Wang, S., Chen, X., Wang, W., Xu, Y., 2017. Three-dimensional evolution of internal waves reflected from a submarine seamount. Phys. Fluids 29 (10), 106601.

Woodson, C., 2018. The fate and impact of internal waves in nearshore ecosystems. Annu. Rev. Mar. Sci. 10, 421-441.

Yuan, C., Grimshaw, R., Johnson, E., Chen, X., 2018a. The propagation of internal solitary waves over variable topography in a horizontally two-dimensional framework. J. Phys. Oceanogr. 48 (2), 283-300.

Yuan, C., Grimshaw, R., Johnson, E., Wang, Z., 2018b. Topographic effect on oblique internal wave-wave interactions. J. Fluid Mech. 856, 36-60.

Zhou, X., Grimshaw, R., 1989. The effect of variable currents on internal solitary waves. Dyn. Atmos. Oceans 14, 17-39. 